\title{
A numerical investigation of laminar forced convection in a solar collector with non-circular duct
}

\author{
Tomasz Janusz Teleszewski ${ }^{1{ }^{* *}}$ \\ ${ }^{1}$ Bialystok University of Technology, Faculty of Civil and Environmental Engineering, Department of \\ HVAC Engineering, ul. Wiejska 45A, 15-351 Bialystok, Poland
}

\begin{abstract}
This paper presents a two-dimensional numerical study to investigate laminar flow in a flat plate solar collector with non-circular duct (regular polygonal, elliptical, and Cassini oval shape) featuring forced convection with constant axial wall heat flux and constant peripheral wall temperature (H1 condition). Applying the velocity profile obtained for the duct laminar flow, the energy equation was solved exactly for the constant wall heat flux using the Boundary Element Method (BEM). Poiseuille and Nusselt numbers were obtained for flows having a different number of geometrical factors. The results are presented and discussed in the form of tables and graphs. The area goodness factor and volume goodness factor are calculated. The predicted correlations for Poiseuille and Nusselt numbers may be a very useful resource for the design and optimization of solar collectors with non-circular ducts.
\end{abstract}

\section{Introduction}

A flat plate solar collector absorbs incoming solar radiation, converting it into thermal energy at the surface of a receiver and heat is transferred to the working fluid through the collector. In fact, the collection of solar radiation and its conversion to thermal energy depend on the collector design, including factors such as riser shape, absorber material, and transmissivity of the glass cover [1]. Simulation of the forced convection in the riser is the most essential part of the process of designing a flat plate collector. The problem of determining the Nusselt number for fully developed laminar flow with triangular cross-section has been solved by Schneider and Ledain [2] using the finite element method to solve the momentum and energy conservation equations. Taherian and Yazdanshenas [3] made an experimental investigation of forced convection in a finned rhombic tube of a flat plate solar collector. Theoretical and experimental investigations of an elliptical heat pipe flat plate solar collector were performed by Sivakumar et al [4]. Forced convection heat transfer in smooth and roughened rectangular ducts has been studied extensively in references [5-9]. Ekramian [1] studied numerically the effect of riser shape on the thermal efficiency, finding a collector with circular tubes, had $38.4 \%, 11.2 \%$, and $6.6 \%$ greater thermal efficiency than collectors with triangular, square, and hexagonal ducts respectively.

*Corresponding author: t.teleszewski@pb.edu.pl 
Fan et al. [10] analysed numerically and experimentally the flow and temperature distribution in a solar collector with a quadrangular absorber tube. Nasrin and Alim [11] numerically simulated forced convection in a cross-section of a flat plate solar collector with nanofluids, using finite elements with Galerkin's weighted residual technique. Dehghan et al. [12] investigated the effects of radiation heat transfer on the forced convective heat transfer mechanism in solar heat exchangers filled with a porous medium. Helvaci and Khan [13] analysed multiphase flow in a flat plate solar energy collector.

The aim of this work is to study the effect of riser shape on the Nusselt number, Poiseuille number, area goodness factor, and volume goodness factor for laminar forced convection, using boundary element method (BEM). These dimensionless quantities may provide the function of screening the selection of a riser shape before other design criteria are applied. There exist varieties of flat plate collector with different non-circular cross-section such as regular polygonal, elliptical, and Cassini oval. The results obtained may lead to the design of more efficient flat plate solar collectors with non-circular ducts.

\section{Mathematical model}

The problem being considered concerns laminar forced convection in solar risers. A simplified numerical model based on the boundary element method for fluid flow and heat transfer in solar risers is developed under the following assumptions: two-dimensional, steady, laminar and constant thermal conductivity $k$ and a constant dynamic viscosity $\mu$ with incompressible fully developed flow, negligible body forces and viscous dissipation, and negligible radiation heat transfer. Newtonian laminar fluid flow is governed by the usual continuity (1), momentum (2) and energy (3) equations for two-dimensional simulations as follows:

$$
\begin{gathered}
\frac{\partial u_{z}}{\partial z}=0 \\
\mu\left(\frac{\partial^{2} u_{z}}{\partial x^{2}}+\frac{\partial^{2} u_{z}}{\partial y^{2}}\right)=\frac{\partial p}{\partial z}, \quad \frac{\partial p}{\partial x}=\frac{\partial p}{\partial y}=0 \\
\lambda\left(\frac{\partial^{2} T}{\partial x^{2}}+\frac{\partial^{2} T}{\partial y^{2}}\right)=\rho c_{p} \frac{\partial T}{\partial z} u_{z}
\end{gathered}
$$

where $u_{z}$ is the axial velocity, $T$ is temperature, $p$ is pressure, $c_{p}$ is specific heat capacity and $\rho$ is density. The coordinate system and computational domain are shown in Figure 1.

In the first step the Poisson equation (2) is solved using the boundary element method. The velocity is required to satisfy the no-slip condition on the duct wall $\left(u_{z}=0\right)$. Secondly, the energy equation (3) is solved for temperature distributions in a cross-section of the duct by BEM. The axial derivative $\partial T / \partial z$ is independent of the $x$ and $y$ coordinates. The energy equation (3) requires the Dirichlet or H1 condition (temperature) and the Neumann or H1 condition (heat flux density), and neglects the axial heat conditions in the fluid. In this paper the H1 condition is considered: $T=$ const, at $L_{h}$ (constant peripheral wall temperature). The $\mathrm{H} 1$ condition has found many applications with negligible normal wall thermal resistance [14], such as in tube heat exchangers and in solar energy collection [4, 13]. Details of the solution of Poisson's equations (2-3) using the boundary element method can be found in the literature [15]. 


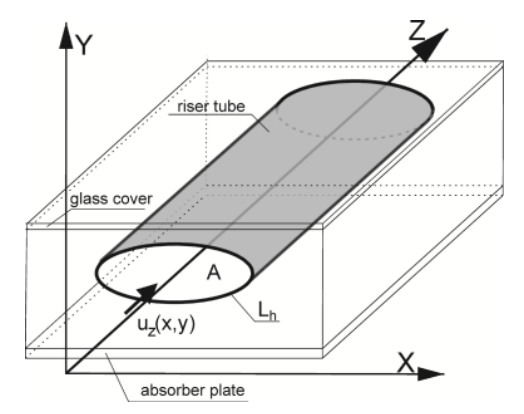

Fig. 1. Schematic diagram of a riser duct in a solar collector.

\section{Analysis and modelling}

The governing equations (2-3) were solved numerically using the boundary element method, by means of a computer program written by the author in Fortran, using 4000 constant boundary elements and 40602 triangular cells in the duct cross-section. To determine relative differences between numerical and analytical values, the numerical results obtained by BEM were compared with the analytical values of the Poiseuille number $\mathrm{Po}$ and the Nusselt number $\mathrm{Nu}$ obtained by Shah and London [14] for a circular tube. For 4000 constant boundary elements and 40602 triangular cells in the duct cross-section, the maximum relative differences in the Poiseuille number and Nusselt number were found as $\Delta \mathrm{Po}=0.001 \%$ and $\Delta \mathrm{Nu}=0.005 \%$.

The Poiseuille number defined as the product of the Fanning friction factor $f$ for fully developed flow and the Reynolds number Re, can be expressed as

$$
P o=f \frac{\operatorname{Re}}{4}, \quad f=\frac{8 \tau_{w}}{\rho u_{m}^{2}}, \quad \mathrm{u}_{m}=\frac{1}{A} \iint_{A} u d A
$$

where $\tau_{w}$ is the peripherally averaged wall shear stress, $u_{m}$ is the mean velocity, and $A$ is the cross-sectional area. The Reynolds number is equal to

$$
\mathrm{Re}=\frac{\rho \mathrm{u}_{m} D_{h}}{\mu}, \quad D_{h}=\frac{4 A}{L_{h}}
$$

where $D_{h}$ is the hydraulic diameter, and $L_{h}$ is the wetted perimeter of the duct cross-section. The Nusselt number is defined as

$$
N u=\frac{q_{w} D_{h}}{k\left(T_{w}-T_{b}\right)}, \quad \mathrm{q}_{w}=\frac{1}{L_{h}} \int_{L_{h}} q d L_{h}
$$

where $T_{w}$ is the wall temperature, $T_{b}$ is the bulk temperature, and $q_{w}$ is the wall heat flux density. In fact, the fully developed laminar Nusselt number is found to be independent of the length of the duct, the Prandtl number Pr and the Reynolds number Re, but dependent on the duct geometry and boundary conditions $[13,14]$.

The considered non-circular geometries include several common shapes, such as regular polygons, ellipses and Cassini ovals. Shah and London [14] provided an analytical relationship for the Poiseuille number and Nusselt number versus the ratio of the minor axis $A$ to the major axis $B$. This analytical relationship contains an elliptic integral of the first kind, which can be computed using numerical integration. Shahsavari et al [16] and 
Teleszewski and Sorko [17] provided numerical relationships for the Poiseuille number and Nusselt number respectively, depending on the number $n$ of sides. No compact relationship has previously been found for the Poiseuille number and the Nusselt number for Cassini ovals. In this paper, a compact relationship for Po and $\mathrm{Nu}$ for ellipses, regular polygons and Cassini ovals versus a geometrical factor $\sigma$, with correlation coefficient $\mathrm{R}^{2} \approx 1$, is proposed:

$$
P o(\sigma), N u(\sigma)=\frac{a+b \sigma^{0.5 m}+c \sigma^{m}+d \sigma^{1.5 m}+e \sigma^{2 m}}{1+f \sigma^{0.5 m}+g \sigma^{m}+h \sigma^{1.5 m}+i \sigma^{2 m}}, \quad \sigma=A / B \vee n \vee C / D
$$

where the coefficients $a, \mathrm{~b}, c, d, e, f, g, h, i, n$ are given in Table 1 . The factor $\sigma$ represents the ratio of the minor to the major axis for an ellipse $(\sigma=A / B)$, the number $\mathrm{n}$ of sides for a regular polygon $(\sigma=n)$, and the ratio of the coefficients $C$ and $D$ in the Cassini oval equation $(\sigma=C / D)$. Equation (7) is more compact and can predict $\mathrm{Po}$ and $\mathrm{Nu}$ values for regular polygonal, elliptical and Cassini oval ducts with an uncertainty less than $0.2 \%$. A Cassini oval is defined as

$$
\begin{aligned}
& \mathrm{x}=\cos (\alpha) \sqrt{\mathrm{C}^{2} \cos (2 \alpha)+\sqrt{\mathrm{C}^{4}(\cos (2 \alpha))^{2}-\mathrm{C}^{4}+\mathrm{D}^{4}}} \\
& \mathrm{y}=\sin (\alpha) \sqrt{\mathrm{C}^{2} \cos (2 \alpha)+\sqrt{\mathrm{C}^{4}(\cos (2 \alpha))^{2}-\mathrm{C}^{4}+\mathrm{D}^{4}}}
\end{aligned}
$$

where $\alpha$ is the angular coordinate.

Table 1. Coefficients of equation (7) to determine the Poiseuille and Nusselt numbers.

\begin{tabular}{|c|c|c|c|c|c|}
\hline \multicolumn{6}{|c|}{ Coefficients of equation (7) to determine the Poiseuille number } \\
\hline shape & $\mathrm{a}$ & $\mathrm{b}$ & $\mathrm{c}$ & $\mathrm{d}$ & $\mathrm{e}$ \\
\hline polygonal & 15.45392 & -27.24564 & 17.31518 & -4.11344 & 0 \\
\hline ellipse & 19.739 & -31.3853 & 74.6744 & -76.4 & 52.576 \\
\hline Cas. oval & 16.012 & -33.43074 & 12.0508 & 12.5435 & -7.17415 \\
\hline & $\mathrm{f}$ & $\mathrm{g}$ & $\mathrm{h}$ & $\mathrm{i}$ & $\mathrm{m}$ \\
\hline polygonal & -1.73499 & 1.0846 & -0.257136 & 0 & 1 \\
\hline ellipse & -1.5874 & 3.7212 & -3.0711 & 2.3876 & 1 \\
\hline Cas. oval & -2.08368 & 0.61023 & 1.05387 & -0.58033 & 4 \\
\hline \multicolumn{2}{|c|}{ Coefficients of equation (7) to determine the Nusselt number } \\
\hline & $\mathrm{a}$ & $\mathrm{b}$ & $\mathrm{c}$ & $\mathrm{d}$ & $\mathrm{e}$ \\
\hline polygonal & -11.1816 & 0 & 2.2561 & 0 & 4.8055 \\
\hline ellipse & 5.2251 & -11.1214 & 31.7343 & -36.338 & 22.0829 \\
\hline Cas. oval & 4.3636 & -14.7687 & 18.6452 & -10.3976 & 2.1576 \\
\hline & $\mathrm{f}$ & $\mathrm{g}$ & $\mathrm{h}$ & $\mathrm{i}$ & $\mathrm{m}$ \\
\hline polygonal & 0 & 0.52438 & 0 & 1.1011 & 1 \\
\hline ellipse & -2.1272 & 6.0374 & -6.3329 & 4.0771 & 1 \\
\hline Cas. oval & -3.39323 & 4.23613 & -2.2834 & 0.440526 & 4 \\
\hline
\end{tabular}


Plots of $\mathrm{Po}$ and $\mathrm{Nu}$ for various values of $\sigma$ are shown in Figures $2 \mathrm{a}$ and $2 \mathrm{~b}$ respectively. Similar trends are observed in both cases. The highest values for $\mathrm{Nu}$ are observed for the Cassini oval with geometrical factor $\sigma=0.945$. In Table 2, the Poiseuille and Nusselt numbers are presented for a Cassini oval duct for some values of the geometrical factor $\sigma=\mathrm{C} / \mathrm{D}$.

a)

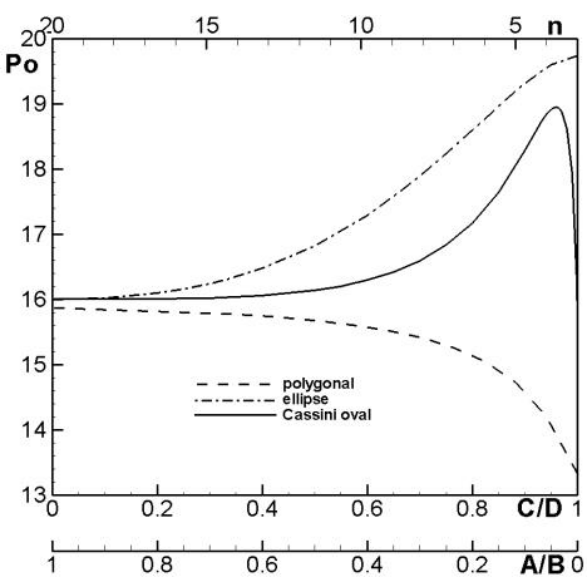

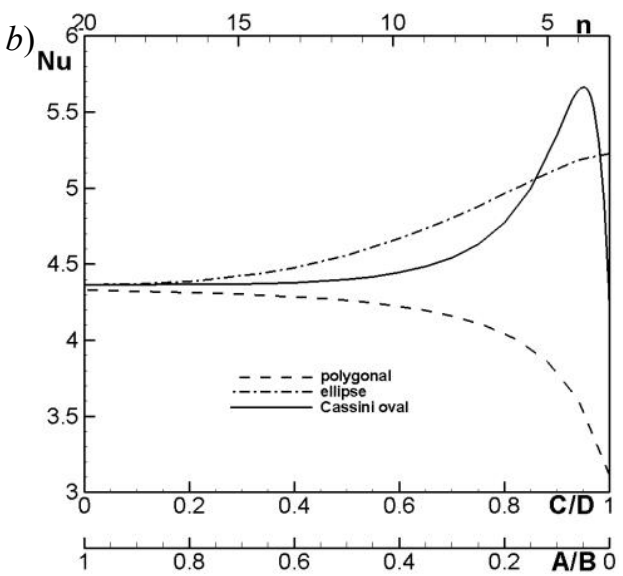

Fig. 2. Plot of $\mathrm{Nu}(\mathrm{a})$ and $\mathrm{Po}(\mathrm{b})$ vs $\sigma$.

The 2D dimensionless velocity $u / u_{m}$ and the dimensionless temperature distribution $T /\left|T_{b}\right|$ with the heatline through the Cassini oval are shown in Figures 3a-b and 4a-b for different values of the geometrical factors: $\sigma=0.85$ and $\sigma=0.945$ respectively. As Figures $3 \mathrm{a}$ and $4 \mathrm{a}$ show, the maximum velocity and minimum fluid temperature occur at the centre of the Cassini oval with $\sigma=0.85$. In Figures $3 \mathrm{~b}$ and $4 \mathrm{~b}$, for a Cassini oval with geometrical factor $\sigma=0.945$, it is interesting to observe that two points of maximum fluid velocity and two points of minimum fluid temperature lie on the axis of symmetry $y=0$.
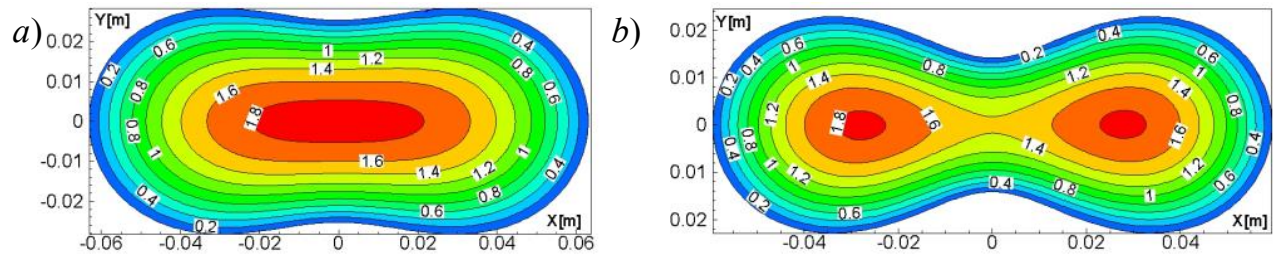

Fig. 3. Dimensionless velocity $\mathrm{u} / \mathrm{u}_{\mathrm{m}}$ obtained from the BEM solution for a Cassini oval duct with different values of $\sigma:(\mathrm{a}) \sigma=0.85(\mathrm{Po}=17.64) ;(\mathrm{b}) \sigma=0.945(\mathrm{Po}=18.88)$.
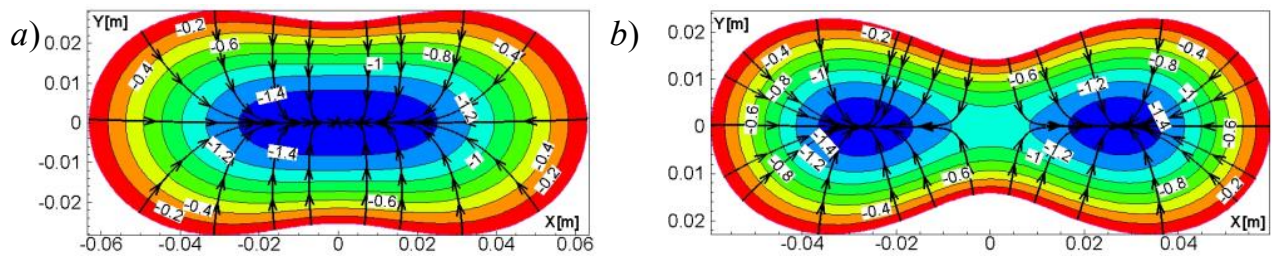

Fig. 4. Heatlines and dimensionless temperature $\mathrm{T} /\left|\mathrm{T}_{\mathrm{b}}\right|$ obtained from the BEM solution for a Cassini oval duct with different values of $\sigma$ : (a) $\sigma=0.85(\mathrm{Nu}=5.00)$; (b) $\sigma=0.945(\mathrm{Nu}=5.66)$. 
Table 2. Poiseuille number and Nusselt number for some values of the factor $\sigma=\mathrm{C} / \mathrm{D}$ for a Cassini oval.

\begin{tabular}{|c|c|c|c|c|c|c|}
\hline \multicolumn{6}{|c|}{ Poiseuille number and Nusselt number vs. $\boldsymbol{\sigma}=$ C/D for Cassini oval } \\
\hline$\sigma=\mathrm{C} / \mathrm{D}$ & 0.2 & 0.4 & 0.6 & 0.8 & 0.945 & 0.98 \\
\hline $\mathrm{Po}$ & 16.01 & 16.06 & 16.30 & 17.18 & 18.88 & 18.60 \\
\hline $\mathrm{Nu}$ & 4.37 & 4.38 & 4.44 & 4.77 & 5.66 & 5.28 \\
\hline
\end{tabular}

\section{Results and discussion}

Two classical types of comparison methods for shaped risers have been considered: area goodness factor [14] and volume goodness factor [14, 18, 19].

In order to compare the thermal performances of riser shapes, it is possible to use the area goodness factor [14], which is the ratio of the Colburn factor $j$ and the Fanning friction factor $f$, defined as:

$$
\mathrm{G}=\frac{j}{f}=\frac{N u}{\operatorname{Pr}^{1 / 3} P o}, \quad \operatorname{Pr}=\frac{c_{p} \mu}{k}
$$

where Pr is the Prandtl number. However, for fully developed forced convection through a straight duct, both the Poiseuille number Po and the Nusselt number are independent of the Prandtl number Pr. Therefore, for this parameter, one may consider only the variations $\operatorname{Pr}^{1 / 3} \mathrm{G}=\operatorname{Pr}{ }^{1 / 3} \mathrm{j} / \mathrm{f}=\mathrm{Nu} / \mathrm{Po}$ as functions of the shape factor. This form of the area goodness factor was introduced by Ray and Misra [20]. The dimensionless area goodness factor is independent of the hydraulic diameter $\mathrm{D}_{\mathrm{h}}$. The area goodness factors of collectors with regular polygonal, elliptical and Cassini oval ducts as functions of the geometrical factor $\sigma$ are presented in Figure 5a. A higher area goodness factor is considered desirable because it means that a lower flow area is required for the riser [14, 21]. Figure 5a shows that the Cassini oval risers with $\sigma=0.945$ have an area goodness factor $28.5 \%, 18.2 \%, 13 \%$ and $9.9 \%$ greater than those of the collectors with triangular, square, elliptical $\sigma=0.1$, and circular riser tubes, respectively. In fact, for the duct geometries having strong corner effects, the area goodness factor is even lower than for channels without sharp corners [14].

a)

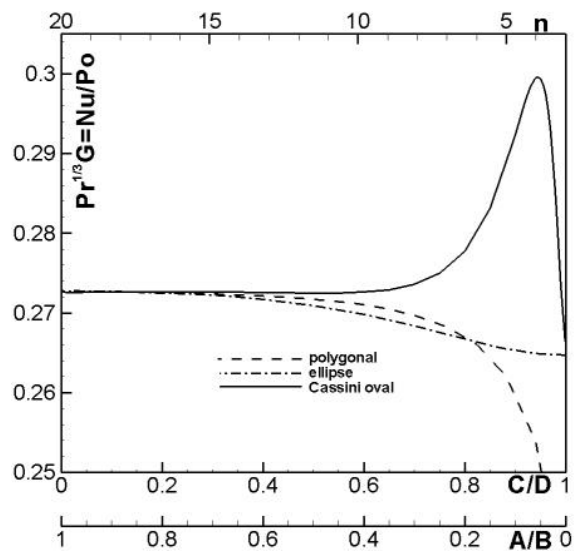

b)

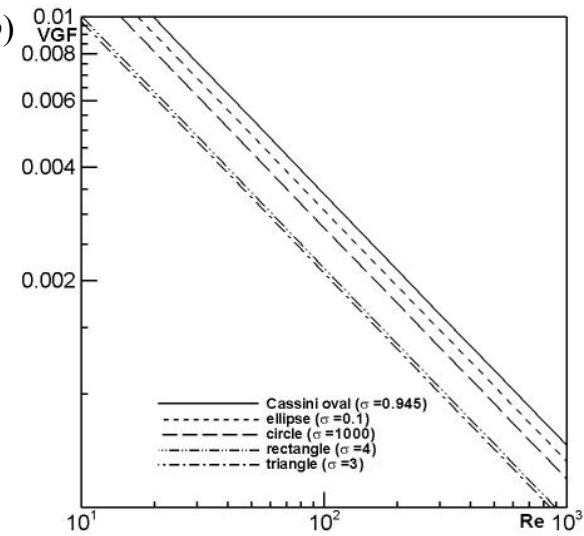

Fig. 5. Comparison methods for riser shape selection: (a) variation of area goodness factor the geometrical parameter factor $\sigma$, (b) volume goodness factor vs. Reynolds number for several riser shapes. 
Another comparison method was suggested by Shah and London [14, 18, 19], using the volume goodness factor $V G F$ as defined by the following equations:

$$
V G F=\frac{\mathrm{St}}{f^{1 / 3}}, \quad \mathrm{St}=\frac{\mathrm{Nu}}{\mathrm{RePr}}
$$

where St is the Stanton number. This factor is used for comparing different heat exchangers $[18,19,21]$. The higher $V G F$ is required the smaller heat transfer. The volume goodness factor is plotted as a function of the Reynolds number in Figure $5 \mathrm{~b}$ for triangular $(\sigma=3)$, square $(\sigma=4)$, elliptical $(\sigma=0.1)$, circular $(\sigma=1000)$ and Cassini oval $(\sigma=0.945)$ risers. The simulations were performed for $50 \%$ propylene glycol mixtures with constant physical quantities $\left(T_{w}=50^{\circ} \mathrm{C}, \mu=0.002\right.$ Pas, $k=0.4 \mathrm{~W} /(\mathrm{mK}), \rho=1025 \mathrm{~kg} / \mathrm{m}^{3}$ and $\left.c_{p}=3480 \mathrm{~J} /(\mathrm{kgK})\right)$. In Figure $5 \mathrm{~b}$ the Cassini oval shape with $\sigma=0.945$ demonstrates higher volume goodness factor than other geometries. Figure $5 \mathrm{~b}$ shows that the Cassini oval risers with $\sigma=0.945$ have an volume goodness factor $62 \%, 57 \%, 10.3 \%$ and $22.7 \%$ greater than those of the collectors with triangular, square, elliptical $\sigma=0.1$, and circular riser tubes, respectively. Plots of the area goodness factor (Figure 5a) and the volume goodness factor (Figure 5b) show that the best performance is obtained for a Cassini oval with geometrical factor $\sigma=0.945$.

Figure 6 is a graphical representation of $\mathrm{Nu}, \mathrm{Nu} / \mathrm{Po}$ and efficiency [1] for regular polygonal ducts versus the number $n$ of sides. In Figure 6, a similar increasing trend is observed for efficiency versus $n$ [1], as for $\mathrm{Nu}$, and $\mathrm{Nu} / \mathrm{Po}$.

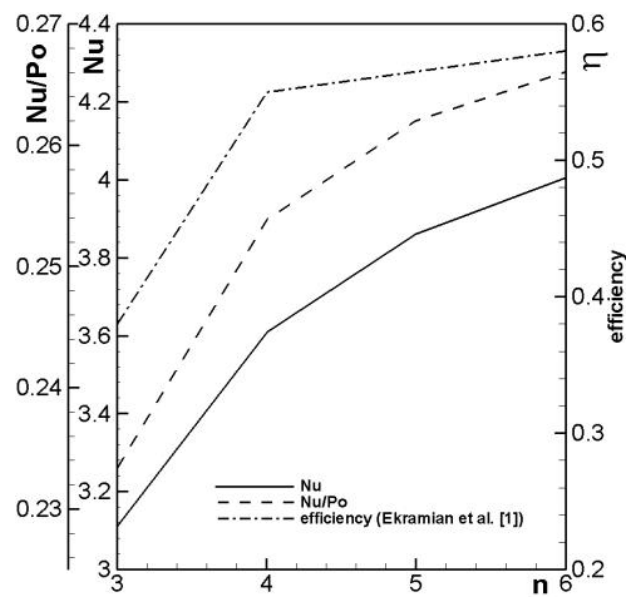

Fig. 6. Plot of $\mathrm{Nu}, \mathrm{Nu} / \mathrm{Po}$, and efficiency [1] vs. $n$ for regular polygon.

\section{Conclusions}

This study has focused on fully developed laminar forced convection in regular polygonal, elliptical and Cassini oval riser ducts in solar collectors. A simple compact correlation between the Poiseuille number and Nusselt number versus a geometrical factor $\sigma$ has been proposed for these riser shapes. The flow is modelled by two-dimensional momentum and energy equations with $\mathrm{H} 1$ boundary conditions. The steady state model has been solved numerically using the boundary element method. This is the first time that a relationship for the Poiseuille number and Nusselt number vs. geometrical shape $(\sigma=C / D)$ has been computed for the Cassini oval. The influence of the analysed non-circular riser shapes on thermal performance was investigated. The results show that the optimum solution for the 
considered non-circular geometries is a Cassini oval with $\sigma=0.945$. The results obtained may be utilized in the optimization of riser geometries.

This scientific project was financed by S/WBiIS scientific research funds at Bialystok University of Technology.

\section{References}

1. E. Ekramian, S.G. Etemad, M. Haghshenasfard, J. of Fluid Flow, Heat and Mass Transfer 1, 1 (2014)

2. G.E. Schneider, B.L. Ledain, J. Energy 5, 1 (1981)

3. H. Taherian, E. Yazdanshenas, In Proceedings of the Second International Green Energy Conference, IGEC2-126 (2006)

4. K. Sivakumar, N.K. Mohan, B. Sivaraman, International J. of Engineering \& Technology 4, 1 (2012)

5. A.M.E. Momin, J.S. Saini, S.C. Solanki, International J. Heat Mass Transfer, 45 (2002)

6. V.S. Hans, R.P. Saini, J.S. Saini, Solar Energy, 84 (2010)

7. S.V. Karmare, A.N. Tikekar, Solar Energy, 84 (2010)

8. A. Kumar, R.P. Saini, J.S. Saini, Renewable Energy, 58 (2013)

9. A. Kumar, Energy Procedia, 57 (2014)

10. J. Fan, L. J. Shah, S. Furbo, Solar Energy, 81 (2007)

11. R. Nasrin, M.A. Alim, J. of Applied Fluid Mechanics 7, 3 (2014)

12. M. Dehghan, Y. Rahmani, D.D. Ganji, S. Saedodin, M. S. Valipour, S. Rashidi, Renewable Energy, 4 (2015)

13. H.U. Helvaci, Z.A. Khan, Energy Conversion and Management, 106 (2015)

14. R.K. Shah, A.L. London, Laminar Flow Forced Convection in Ducts, Advanced in Heat Transfer (Academic Press, London, UK, 1978)

15. C.A. Brebbia, J.C.F. Telles, L.C. Wrobel, Boundary Element Techniques-Theory and Applications in Engineering (Springer-Verlag, Berlin, Heidelberg, New York, Tokyo, 1984)

16. S. Shahsavari, A. Tamayol, E. Kjeang, M. Bahrami, ASME J. of Heat Transfer 134, 9 (2012)

17. T.J. Teleszewski, S.A. Sorko, Procedia Engineering, 157 (2016)

18. J.-H. Doo, H.S. Yoon, M.Y. Ha, Transactions of the Korean Society of Mechanical Engineers B 33, 6 (2009)

19. S.W. Hwang, D.H. Kim, J.K. Min, J.H. Jeong, Journal of Mechanical Science and Technology 26, 9 (2012)

20. S. Ray, D. Misra, Int. J. Therm. Sciences, 49 (2010)

21. K.M. Stone, ACRC TR-105 (1996) 